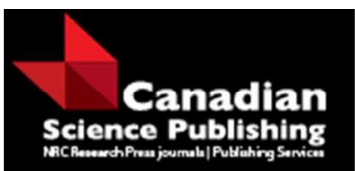

Canadian Journal of Forest Research

Revue canadienne de recherche forestière

\title{
Dimensionless numbers for the sustainable harvesting of a monospecific uneven-aged forest
}

\begin{tabular}{|r|l|}
\hline Journal: & Canadian Journal of Forest Research \\
\hline Manuscript ID: & cjfr-2015-0140.R2 \\
\hline Manuscript Type: & Article \\
\hline Date Submitted by the Author: & 29-Jun-2015 \\
\hline Complete List of Authors: & $\begin{array}{l}\text { L pez Torres, Ignacio; Universidad Polit cnica de Madrid } \\
\text { Fullana Belda, Carmen; Universidad Pontificia de Comillas }\end{array}$ \\
\hline Keyword: & $\begin{array}{l}\text { Dimensionless numbers, Equilibrium, Sustainable harvesting, Matrix model, } \\
\text { Stability }\end{array}$ \\
\hline \multicolumn{2}{|l}{} \\
\hline
\end{tabular}

\section{SCHOLARONE \\ Manuscripts}




\section{Dimensionless numbers for the sustainable harvesting of a monospecific uneven-aged forest}

4

5

6

7

8

9

10

1

12

13

14

15

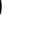

Keywords: Dimensionless numbers; Equilibrium; Sustainable harvesting; Matrix model; Natural disturbances.

Running Title: Dimensionless numbers for sustainable harvesting. 


\section{1. Introduction}

35 In fluid mechanics and aerodynamics, the Reynolds number is a dimensionless number computed as

36 the ratio of inertial forces to viscous forces, that quantifies the relative importance of these two

37 forces for given flow conditions. A critical value of this number (in general experimentally

38 determined, and that should not be interpreted narrowly) is often used to estimate the transition

39 between laminar and turbulent flow.

40 Similarly, in hydrology and fluid dynamics, the Froude number is a dimensionless number defined as

41 the ratio of inertial to gravitational forces, which can describe different flow regimes. In free-surface

42 flow, the nature of the flow, supercritical (fast flow) or subcritical (slow flow), depends upon

43 whether the Froude number is greater than or less than unity.

44 In general, dimensionless numbers (which are numbers representing a property of a system, but

45 without any physical units, such as length $L$, area $L^{2}$, mass, or time, associated with them), help in the

46 understanding of certain problems for which no analytic mathematical formulation exists, or for

47 which the mathematical formulation is too complex to be easily comprehended. In case of existence

48 of such numbers for a specific system, they allow to extract certain conclusions about the behavior

49 of the system without the need of a more detailed mathematical formulation relating all the

50 different variables involved in the problem at hand. Critical values of these numbers are important

51 to identify bifurcations between different states of the system. Thus, they are widely used in many

52 fields, such as mathematics, physics, biology, engineering, and economics (e.g. see Szirtes (2006)).

53 In this study, we demonstrate that in the particular case of uneven-aged monospecific stand

54 dynamics, where we can also find a "demographic flow" (defined by the stems evolution through the

55 diameter classes over time), there are dimensionless numbers which have a strong influence on the

56 behaviour of the system. Moreover, critical values of those numbers identify a bifurcation in the

57 stand management practices switching between sustainable and unsustainable harvesting. 
58 The main factors influencing this flow (in the absence of external disturbances) are recruitment, 59 transition probabilities between diameter classes, and mortalities (natural or induced by harvesting) 60 (Schütz, 2006). These processes of evolution over time can easily be modeled by a matrix population 61 model (e.g. Buongiorno and Michie, 1980; Liang and Picard, 2012; Vanclay, 2012) for which the 62 population growth rate is the dominant eigenvalue $\lambda$ of the transition matrix. By asymptotic analysis (long-term behaviour), we know that (Caswell, 2001), independent of the initial conditions, when $\lambda>1$ the total number of stems/ha of the tree population increases exponentially over time (unless harvests are conducted), when $\lambda<1$ the population is decaying until extinction, and when $\lambda=1 \mathrm{a}$ stable distribution proportional to the right eigenvector $W_{0}$ of the transition matrix corresponding to $\lambda$ is obtained. Gotelli (2001) refers to the special case of the stable distribution when $\lambda=1$ as the "stationary distribution"; we consider the same case in reference to the stable diameter distribution of the stand $W_{0}$.

Therefore, in this study we have used the matrix model applied to uneven-aged managed Fagus sylvatica L. and Pinus nigra stands by López et al. (2007, 2008, 2012, 2013), which describes accurately the stand dynamics. In particular, in order to exemplify and test the results, the model uses the transition probabilities obtained in López et al. $(2012,2013)$ for uneven-aged managed Pinus nigra stands, considering three levels of tree diameter growth (Quality I, faster; Quality II, medium; and Quality III, slower diameter growth) (see Table 1). The stands are located in the Spanish Iberian System, a mountain range extending about $400 \mathrm{~km}$ along the north-eastern edge of the central plateau, concentrating $60 \%$ of the area occupied by Pinus nigra in Spain (Grande et al. 2005). Based on data from those stands, the model simulates outcomes for 594 different forest management planning scenarios: 3 diameter growth levels (Qualities I-III) $\times 6$ basal area levels $(G=$ 21 to $\left.G=26 \mathrm{~m}^{2} / \mathrm{ha}\right) \times 33$ recruitment levels $(R=200$ to $R=840$ stems $/ \mathrm{ha})$. 
83

84

85

\section{Methods}

\subsection{Matrix model}

The matrix model for the recruitment, harvesting and stem flow throughout the dbh-classes over time is defined by means of a finite difference homogeneous linear system of equations, which accurately describes transitions from time $t$ (initial) to time $t+1$ (final). Since the harvesting operations in the study area generally took place every 10 years, this was the time step adopted in the model as an example (thus the time interval $(t, t+1)$ represents 10 years). Considering this time step, trees were grouped into $n$ diameter classes of equal width $w=6 \mathrm{~cm}$ (the parameter $w$ represents the width of the diameter classes): $(0,6],(6,12],(12,18], \ldots,(30,36], \ldots$, (i.e., $(6,12]$ means a diameter interval which contains the diameter $12 \mathrm{~cm}$ but not the diameter $6 \mathrm{~cm}$ ), with the last class being $(48, \rightarrow)$ (more than $48 \mathrm{~cm}$ ) for Quality I, $(42, \rightarrow)$ (more than $42 \mathrm{~cm}$ ) for Quality II and $(36, \rightarrow)$ (more than $36 \mathrm{~cm}$ ) for Quality III. Therefore, an individual tree in class $k$ can remain in class $k$ or progress to class $k+1$ during the projection interval $(t, t+1)$. The number of trees in each class changes in each projection interval, because some are harvested, some remain in the same diameter class and others grow past the boundary to the next diameter class. In such conditions, $p_{k}$ is the probability that an individual tree in class $k$ at time $t$ (initial) will appear in class $k+1$ at time $t+1$ (final time of projection); $r_{k}$, recruitment coefficient, is the number of offspring (stems/ha) living at time $t+1$ produced in the interval of projection $(t, t+1)$ by an average tree in class $k$ at time $t$ (similar to many standard size classified matrix models (Caswell, 2001), $\left.r_{1}=0\right) ; h_{k}(t)$ defines the proportion of harvested trees in class $k$, natural mortalities included; $x_{k}(t)$ and $x_{k}(t+1)$ describe the stem densities in class $k$ at the initial and final times of projection. Finally, $R=r_{2} x_{2}+r_{3} x_{3}+\ldots+r_{n} x_{n}$ will denote the global amount of recruitment at each time step. Notice that $0<p_{k}<1$ for $k=1,2, \ldots, n-1 ; r_{k} \geq 0$ for $k=2,3, \ldots, \mathrm{n}$, with $R=0$ if and only if $r_{k}=0$ for all $k$; and $x_{k}>0$ for $k=1,2, \ldots, n$.

By analysing the dynamics of the projections, we find that the model is described accurately by the matrix model 


$$
X(t+1)=A(I-H(t)) X(t)
$$

where

$$
A=\left(\begin{array}{cccccc}
1-p_{1} & r_{2} & r_{3} & \cdots & r_{n-1} & r_{n} \\
p_{1} & 1-p_{2} & 0 & \cdots & 0 & 0 \\
0 & p_{2} & 1-p_{3} & \cdots & 0 & 0 \\
\vdots & \vdots & \vdots & \ddots & \vdots & \vdots \\
0 & 0 & 0 & \cdots & 1-p_{n-1} & 0 \\
0 & 0 & 0 & \cdots & p_{n-1} & 1
\end{array}\right)
$$

$111 I$ is the identity matrix of order $n, H(t)=\operatorname{diag}\left(h_{1}(t), h_{2}(t), \ldots, h_{n}(t)\right)$ is a diagonal matrix with the

112 harvest rates $h_{k}(t)$ (including natural mortalities), and $X(t)$ and $X(t+1)$ are column vectors indicating 113 the stem densities at the initial and final times of projection, respectively. Note that the model only supports transitions between consecutive diameter classes. Therefore, the time step and the width of the diameter classes should be chosen according to this prerequisite.

\subsection{Sustainable/stable harvesting strategy}

As we can see in (1), harvesting induces a perturbation in the model of natural growth of trees (i.e. growth model without harvesting), defined by

$$
X(t+1)=A X(t)
$$

modifying the natural transition matrix $A$ toward the perturbed $A(I-H)$. Thus, whenever the dominant eigenvalue of matrix $A$ be $\lambda_{0}>1$, the long-term sustainable harvest rates can be determined as the proportion of trees harvested in each class so that the dominant eigenvalue of matrix $A(I-H)$ be $\lambda=1$. There are many different harvesting strategies to satisfy this boundary condition $\lambda=1$, and many other unable to achieve this condition. In particular, the et al., 2007, 2008): 
129 (C3) Each harvest should lead to the stable diameter distribution of the stand, which is given by the 130 right eigenvector $W_{0}$ corresponding to the population growth rate $\lambda_{0}$ of the matrix $A$.

131 Note that $\lambda_{0} \geq 1$, and $\lambda_{0}=1$ for $R=0$. In fact, from $\lambda_{0}=1$ for $R=0$ (as we can see in the matrix $A$ for $\left.132 r_{2}=r_{3}=\ldots=r_{n}=0\right)$, and $\frac{\partial \lambda_{0}}{\partial R} \geq 0$, as we know by sensitivity analysis (Caswell, 1978), it follows that $133 \lambda_{0} \geq 1$.

Moreover, assuming that condition (C1) holds, conditions (C2) and (C3) can be rewritten by means of the linear system $A H W_{0}=\left(\lambda_{0}-1\right) W_{0}$. Thus, we obtain the "sustainable/stable" harvest rates $s$ by solving this determinate compatible linear system, yielding the following rate

$$
s=\frac{\lambda_{0}-1}{\lambda_{0}}
$$

138 for all the diameter classes. In this equation (3), $s^{\prime}=\frac{1}{\lambda_{0}}=1-s$ has the interpretation of the proportion of trees that has to remain unharvested (for all the diameter classes) to retain the stable diameter distribution (with sustainability, since $\lambda=1$ ).

Let us suppose a harvesting strategy such that $h_{1}=h_{2}=\ldots=h_{n-1}=h_{n}=h$, with $h<s$. Then, $\lambda>1$, and $h$ is a sustainable harvest rate, although the resources are under harvested and the stable diameter distribution of the stand is not reached at each time step. Conversely, if $h_{1}=h_{2}=\ldots=h_{n-1}=h_{n}=h$, with $h>s$, then, $\lambda<1, h$ is not a sustainable harvest rate and the harvest does not lead to the stable diameter distribution of the stand either. Hence, the "sustainable/stable" harvest rate $s$ (and, by extension, $s^{\prime}$ ) defines a boundary between sustainable and unsustainable harvesting.

It would therefore be useful to find easy expressions, involving the main variables of the problem, which help identify this bifurcation between sustainable and unsustainable harvesting. For this we will manipulate the equation for the dynamic stand equilibrium introduced by López et al. (2007) and generalized in López et al. (2008). 
151

152

153

154

155

156

157

158

159

160

161

162

163

164

165

166

167

\subsection{Equilibrium equation. Dimensionless numbers}

Let us consider first the growth model without harvesting (2), then the harvesting model (1), but bearing in mind that both models are related in the bifurcation corresponding to the "sustainable/stable" harvesting strategy by means of their transition matrices

$$
A(I-H)=(1-s) A
$$

From (4) it follows that the corresponding population growth rates $\lambda_{0}$ and $\lambda$ are related by the equation

$$
\lambda=(1-s) \lambda_{0}
$$

We now recall (López et al., 2007, 2008) that, by solving $A W_{0}=\lambda_{0} W_{0}$, we obtain the components of the stable diameter distribution of the stand $W_{0}$ (which is simultaneously the right eigenvector of the matrices $A$ and $A(I-H)$ corresponding respectively to the dominant eigenvalues $\lambda_{0}$ and $\lambda=1$ ), yielding (proportional to) the vector $W_{0}=\left(N_{1}, N_{2}, N_{3}, \ldots, N_{n-1}, N_{n}\right)$, where

$$
\begin{gathered}
N_{1}=\left(\lambda_{0}-1\right) \prod_{k=2}^{n-1}\left(\lambda_{0}-1+p_{k}\right), N_{i}=\left(\lambda_{0}-1\right)\left(\prod_{k=1}^{i-1} p_{k}\right)\left(\prod_{k=i+1}^{n-1}\left(\lambda_{0}-1+p_{k}\right)\right) \text { for } 1<i<n-1, \\
N_{n-1}=\left(\lambda_{0}-1\right) \prod_{k=1}^{n-2} p_{k}, \text { and } N_{n}=\prod_{k=1}^{n-1} p_{k} .
\end{gathered}
$$

By dividing consecutive components, we arrive at

$$
N_{i+1}=\frac{p_{i}}{\lambda_{0}-1+p_{i+1}} N_{i}=l_{i} N_{i}, \text { for } i=1,2, \ldots, n-2, \text { and } N_{n}=\frac{p_{n-1}}{\lambda_{0}-1} N_{n-1}=l_{n-1} N_{n-1}
$$

where $l_{i}=\frac{p_{i}}{\lambda_{0}-1+p_{i+1}}$, for $i=1,2, \ldots, n-2$ and $l_{n-1}=\frac{p_{n-1}}{\lambda_{0}-1}$.

Substituting $N_{i}$ (for $i=1,2, \ldots, n-1, n$ ) from (6) into the equation of the basal area of the stand $G$ (prior to harvest), we get 


$$
\left(1-p_{1}\right) N_{1}+\sum_{i=2}^{n} r_{i} N_{i}=\left(1-p_{1}\right) N_{1}+R=\lambda_{0} N_{1}
$$

$$
N_{1}=\frac{R}{\lambda_{0}-1+p_{1}}
$$

$$
G=\frac{\pi R}{4\left(\lambda_{0}-1+p_{1}\right)}\left[\frac{w^{2}}{4}+\sum_{i=2}^{n}\left(\frac{(2 i-1)^{2} w^{2}}{4} \prod_{k=1}^{i-1} l_{k}\right)\right] .
$$

$$
P_{n}\left(\lambda_{0}\right)=a_{n}\left(\lambda_{0}-1\right)^{n}+a_{n-1}\left(\lambda_{0}-1\right)^{n-1}+a_{n-2}\left(\lambda_{0}-1\right)^{n-2}+\ldots+a_{1}\left(\lambda_{0}-1\right)+a_{0}=0
$$

We recall that the dominant (positive) root of this polynomial $P_{n}\left(\lambda_{0}\right)=a_{n}\left(\lambda_{0}-1\right)^{n}+\ldots+a_{1}\left(\lambda_{0}-1\right)+a_{0}$ is

The coefficients $a_{k}$ of the polynomial $P_{n}\left(\lambda_{0}\right)$ are specified in Appendix 1 in the general case, and in

$$
a_{n}=\frac{16 G}{\pi w^{2} R}
$$




$$
a_{0}=-(2 n-1)^{2} \prod_{i=1}^{n-1} p_{i}
$$

have a strong influence on $\lambda_{0}$. In fact, by applying the Descartes' rule of signs, considering that the number of changes of sign in the list of coefficients of $P_{n}\left(\lambda_{0}\right)$ was 1 for any of the 594 examined scenarios (with $a_{n}>0$ and $a_{0}<0$ in any case), $P_{n}\left(\lambda_{0}\right)$ has exactly one (positive) root greater than 1 .

Moreover, a rough approximation $\lambda_{0}$ of the root $\lambda_{0}$ may be obtained from

$$
P_{n}\left(\lambda_{0}\right)=a_{n}\left(\lambda_{0}-1\right)^{n}+a_{0}=0
$$

yielding

$$
\lambda_{0} \approx \lambda_{0}=1+\sqrt[n]{-\frac{a_{0}}{a_{n}}}=1+\sqrt[n]{\frac{(2 n-1)^{2} \pi w^{2} R \prod_{i=1}^{n-1} p_{i}}{16 G}}
$$

Therefore, a dimensionless number for the growth model without harvesting (2) is given by

$$
\lambda_{0}=1+\sqrt[n]{\frac{(2 n-1)^{2} \pi w^{2} R \prod_{i=1}^{n-1} p_{i}}{16 G}}
$$

As equation (14) suggests and we show in Section 3 , the variables $\lambda_{0}$ and $\lambda_{0}$ have a strong positive linear correlation.

Regarding the growth model with harvesting (1), a rough approximation $\hat{\lambda}$ of the root $\lambda=1$ corresponding to the "sustainable/stable" harvesting strategy may be obtained from (5) and (15). In fact, as we show in Section 3, the dimensionless number $\hat{\lambda}=(1-s) \chi_{0}$, 


$$
\hat{\lambda}=(1-s)\left(1+\sqrt[n]{\frac{(2 n-1)^{2} \pi w^{2} R \prod_{i=1}^{n-1} p_{i}}{16 G}}\right)=\frac{\lambda_{0}}{\lambda_{0}},
$$

202

203

204 is very close to 1 for any of the 594 examined scenarios. Therefore, for a harvesting strategy such that $h_{1}=h_{2}=\ldots=h_{n-1}=h_{n}=h$, with $h<s$, we would obtain

$$
(1-h)\left(1+\sqrt[n]{\frac{(2 n-1)^{2} \pi w^{2} R \prod_{i=1}^{n-1} p_{i}}{16 G}}\right)>1,
$$

thus $h$ is a sustainable harvest rate. Conversely, if $h_{1}=h_{2}=\ldots=h_{n-1}=h_{n}=h$, with $h>s$, then

$$
(1-h)\left(1+\sqrt[n]{\frac{(2 n-1)^{2} \pi w^{2} R \prod_{i=1}^{n-1} p_{i}}{16 G}}\right)<1
$$

so $h$ is not a sustainable harvest. Hence, the dimensionless number $\hat{\lambda}$ defines a boundary between sustainable (equation (17)) and unsustainable (equation (18)) harvesting. However, since $s$ (and, by extension, $\lambda_{0}$ ) is needed for calculating $\hat{\lambda}$, it would be more useful to find another boundary, which is not based on those variables. This can be done by considering equations (16), (17) and (18), from which we define a rough approximation $S$ to the "sustainable/stable" harvest rate $s$ by means of

$$
(1-\hat{s})\left(1+\sqrt[n]{\frac{(2 n-1)^{2} \pi w^{2} R \prod_{i=1}^{n-1} p_{i}}{16 G}}\right)=1,
$$




$$
\hat{s}=1-\left(1+\sqrt[n]{\frac{(2 n-1)^{2} \pi w^{2} R \prod_{i=1}^{n-1} p_{i}}{16 G}}\right)^{-1}=\frac{\lambda_{0}-1}{\lambda_{0}}
$$

215 In this case, a harvesting strategy such that $h_{1}=h_{2}=\ldots=h_{n-1}=h_{n}=h$, with $h<\hat{S}$, should be

216 sustainable and, conversely, if $h_{1}=h_{2}=\ldots=h_{n-1}=h_{n}=h$, with $h>\hat{S}$, then the harvesting strategy

217 should be unsustainable.

Nevertheless, for reasons which will be explained in Section 3, in general it would be better to use in

219 this case $s^{\prime}$, rather than $\hat{s}$, defined as a rough approximation to $s^{\prime}=\frac{1}{\lambda_{0}}=1-s$ (proportion of trees

220 that has to remain unharvested to retain the stable diameter distribution) given by

$$
s^{\prime}=1-\hat{s}=\frac{1}{\lambda_{0}}=\left(1+\sqrt[n]{\frac{(2 n-1)^{2} \pi w^{2} R \prod_{i=1}^{n-1} p_{i}}{16 G}}\right)^{-1} .
$$

Finally, as we show in Section 3, a rough approximation $W_{0}$ to the stable diameter distribution of the

$$
N_{1}=\left(\lambda_{0}-1\right) \prod_{k=2}^{n-1}\left(\lambda_{0}-1+p_{k}\right), \lambda_{i}=\left(\lambda_{0}-1\right)\left(\prod_{k=1}^{i-1} p_{k}\right)\left(\prod_{k=i+1}^{n-1}\left(\lambda_{0}-1+p_{k}\right)\right) \text { for } 1<i<n-1,
$$

$$
\AA_{n-1}=\left(\chi_{0}-1\right) \prod_{k=1}^{n-2} p_{k}, \text { and } N_{n}=\prod_{k=1}^{n-1} p_{k}
$$




\section{8 \\ 2.4. Extension to the case of natural perturbations.}

229 In the context of matrix models, natural disturbances can be addressed in several ways (see Liang et

230 al., 2005; Liang and Picard, 2012), and abundant evidence suggests that considering natural

231 perturbations can make huge differences in stand dynamics and forest equilibrium (e.g. Zhou and

232 Buongiorno, 2004). In a broad sense, a system is considered to be stable if it always returns to a

233 reference position (equilibrium) after small perturbations (i.e., natural disturbances in forests). As

234 shown in the Introduction, in the case of the discrete dynamic system (2), we say that the

235 equilibrium position defined by $W_{0}$ is stable because the trajectories (that is, solutions represented

236 in the phase space, where all possible states of the system are represented) with initial condition

237 selected in a sufficiently small neighborhood of that equilibrium, always remain near the

238 equilibrium.

239 However, in applications of Perturbations Theory and Structural Stability of Dynamical Systems (see,

240 for example, Hirsch and Smale (1974)), we require our mathematical model to be more robust (not

241 just stable equilibrium). By this we mean that its qualitative properties (geometric shape of

242 trajectories in the phase space, type of stability of equilibrium points and critical solutions, etc.)

243 should not change significantly when the model is subjected to small perturbations (i.e., small

244 changes on the right hand side of the system of linear difference equations (2)). In such a case, we

245 say that the system is structurally stable. In particular, if (2) is structurally stable and it has an

246 attractor (as the stable diameter distribution $W_{0}$ ), any nearby perturbed system will have a nearby

247 attractor (we say that the attractor persists).

248 A simple necessary and sufficient condition for the structural stability of a linear dynamical system is

249 that all the eigenvalues of the associated matrix have non-zero real parts. In general, this condition is

250 easily met in the case of linear systems of difference equations modeling forest dynamics, in

251 particular in the equation (2) for all scenarios. 
252 In our study, the perturbation on the model of natural growth of trees (2) due to harvesting, is given 253 by

255

256

257

258

259

260

261

262

263

264

where

$$
H=\operatorname{diag}\left(h_{1}, h_{2}, \ldots, h_{n}\right)
$$

is a diagonal matrix with the harvest rates (including natural mortalities) for each time step (see also equation (1)). Since all the eigenvalues of the matrix $A$ have non-zero real parts, $(2)$ is structurally stable. In particular, the attractor given by the stable diameter distribution is persistent under small perturbations caused by harvesting.

Note that (22) is a linear perturbation of system (2), which is structurally stable. Let us now prove that the results from Sections 2.2. and 2.3. can be extended to the case of any linear perturbation $E$ of (2), defined by

where $\varepsilon_{i}$ represents the perturbation rate for the ith diameter class, comprising harvest rates $\left(\mu_{i}\right)$ (excluding in this case natural mortalities) and natural disturbance rates $\left(\delta_{i}\right)$ (natural mortalities included), that is, $\varepsilon_{i}=\mu_{i}+\delta_{i}$. In fact, the model (2) of natural growth of trees including natural perturbations is defined by means of

$$
X(t+1)=A(I-\Delta) X(t)
$$

where $\Delta=\operatorname{diag}\left(\delta_{1}, \delta_{2}, \ldots, \delta_{n}\right)$ is a diagonal matrix with the natural disturbance rates. The model of natural growth of trees including natural perturbations and harvesting is given by

$$
X(t+1)=A(I-\Delta-H) X(t)
$$

where $H=\operatorname{diag}\left(\mu_{1}, \mu_{2}, \ldots, \mu_{n}\right)$ is a diagonal matrix with the harvest rates. Let $\lambda_{0}$ be the dominant eigenvalue of matrix $A(I-\Delta)$, with $\lambda_{0}>1$ (otherwise condition (C1) is not verified), and $W_{0}$ the 
275

276

277

278

279

280

281

282

283

284

285

286

287

288

290

291

292

293

associated eigenvector (stable diameter distribution corresponding to the matrix $A(I-\Delta)$ ). By substituting $h_{i}$ for $\mu_{i}$ in (22), conditions (C2) and (C3) can be rewritten by means of the linear system

$$
A \operatorname{diag}\left(\mu_{1}, \mu_{2}, \ldots, \mu_{n}\right) W_{0}=\left(\lambda_{0}-1\right) W_{0}
$$

Since $A W_{0} \approx \lambda_{0} W_{0}$ (let us recall that (23) is the perturbed system of (2) and that the attractors of structurally stable systems persist under small perturbations), a fairly good approximation to the solution of this system is the "sustainable/stable harvest rate" $\mu$ (natural perturbations excluded), defined by means of

$$
\mu=\frac{\lambda_{0}-1}{\lambda_{0}}
$$

for all the diameter classes (that is, $\mu_{1}=\mu_{2}=\ldots=\mu_{n}=\mu$ ). Note that in this equation $\lambda_{0}$ is assuming the natural disturbances $\delta_{i}$ from the matrix $A(I-\Delta)$ (internally, since $\delta_{i}$ form part of the coefficients of the matrix $A(I-\Delta))$. Moreover, the results of section 2.3. can be easily extended by carrying out the following changes:

1. Throughout Section 2.3. the transition probabilities are given by $p_{k}\left(1-\delta_{k}\right)$.

2. By solving (8) for $N_{1}$ (considering the model (23)), we obtain

$$
N_{1}=\frac{R-M_{R}}{\lambda_{0}-\left(1-p_{1}\right)\left(1-\delta_{1}\right)},
$$

where $M_{R}=\delta_{2} r_{2} x_{2}+\delta_{3} r_{3} x_{3}+\ldots+\delta_{n} r_{n} x_{n}$ is the global mortality for tree recruitment due to natural disturbances (stems/ha). We recall that $\lambda_{0}$ is the dominant eigenvalue of matrix $A(I-\Delta)$.

3. The equilibrium equation (9) for the model (23) becomes

$$
G=\frac{\pi\left(R-M_{R}\right)}{4\left(\lambda_{0}-\left(1-p_{1}\right)\left(1-\delta_{1}\right)\right)}\left[\frac{w^{2}}{4}+\sum_{i=2}^{n}\left(\frac{(2 i-1)^{2} w^{2}}{4} \prod_{k=1}^{i-1} l_{k}\right)\right]
$$


assuming in $I_{k}$ the new transition matrix coefficients.

4. The expressions (11) and (12) for the model (23) are given by

$$
a_{0} \approx-(2 n-1)^{2} \prod_{i=1}^{n-1} p_{i}\left(1-\delta_{i}\right)
$$

(the equality for $a_{0}$ holds if $\delta_{n}=0$ ).

5. The expressions (11) to (20) should be adapted to include the new $a_{0}$ and $a_{n}$ given by (24) and 300

301 (25). For example, the expression (14) becomes

(the equality holds if $\delta_{n}=0$ ).

303

6. Renaming and redefining variables, $s$ as $\mu, \hat{s}$ as $\mu$, and $s^{\prime}$ as $\mu^{\prime}$ (defined by $\mu^{\prime}=1-\mu$ ). For 304 example,

305

$$
\mu=1-\left(1+\sqrt[n]{\frac{(2 n-1)^{2} \pi w^{2}\left(R-M_{R}\right) \prod_{i=1}^{n-1} p_{i}\left(1-\delta_{i}\right)}{16 G}}\right)^{-1}=\frac{\lambda_{0}-1}{\lambda_{0}} .
$$

306 The natural mortality rate was included into the harvesting rate in Section 2.1. considering that all 307 dead trees along the decade are harvested subsequently, but it's clear that for higher stand densities than the values considered in our scenarios, the natural disturbances rate (natural mortalities 
309 included) could be high, in particular for the lower diameter classes. . In such cases, it should be used

310 the model (23) and the associated results.

311 With regard to nonlinear perturbations, the results from Sections 2.2. and 2.3. cannot be directly 312 extended, and each case must be analyzed individually. However, the strong property of structural 313 stability of system (2) may be applied, obtaining a first order approximation to the solutions of the 314 (nonlinear) perturbed system of (2) by means of the corresponding linearization, which might be 315 addressed in the same manner as the linear perturbation $E$ described above.

\section{3. Results}

Since the results from Sections 2.2. and 2.3. can be easily extended to the case of the linear perturbation introduced in Section 2.4., simulations were conducted using only the first case. By substituting the transition probabilities $p_{i}$ from Table 1 into (10) and solving the corresponding polynomial equation for each scenario, we obtained the population growth rate $\lambda_{0}$ and, subsequently, the "sustainable/stable" harvest rate $s$ (equation (3)). Moreover, by substituting $p_{i}$, recruitment $R$, basal area $G$, together with $w$ and $n$, into the equations (15), (16), (19) and (20), we

323 obtained $\boldsymbol{\lambda}_{0}, \hat{\lambda}, \hat{S}$ and $s^{\prime}$, as summarized in Tables 2, 3 and 4 for selected scenarios.

324 The relative error between $\lambda_{0}$ and $\lambda_{0}$, defined as

$$
\eta_{\lambda_{0}}=\frac{\lambda_{0}-\lambda_{0}}{\lambda_{0}}=1-\hat{\lambda},
$$

ranged in absolute value from $1.85 \%$ to $19.42 \%$ for Quality I (averaging $9.66 \%$ ), from $2.47 \%$ to 17.47\% for Quality II (averaging 9.24\%), and from 1.89\% to 14.45\% for Quality III (averaging $7.66 \%$ ),

328 always with $\lambda_{0}<\lambda_{0}$. The maximum relative errors between $\lambda_{0}$ and $\lambda_{0}$, occurred in any case for the 
329

330

332

335 we note that, while $\eta_{s}$ expands the error $\eta_{\vartheta^{\prime}}, \eta_{s^{\prime}}$ reduces it by a factor of $\left|\frac{1}{\eta_{\lambda_{0}}-1}\right|<1$. In fact, the $G=26 \mathrm{~m}^{2} /$ ha and $R=200$ stems $/$ ha scenarios, and the minimum for the $G=21 \mathrm{~m}^{2} /$ ha and $R=840$ stems/ha scenarios. Between $\lambda$ (with $\lambda=1$ ) and $\hat{\lambda}$ we have the same relative errors, because

$$
\eta_{\lambda}=1-\hat{\lambda}=\eta_{\lambda_{0}}
$$

With regard to the relative error between $s$ and $\hat{S}, s^{\prime}$ and $\hat{S}^{\prime}$, defined respectively as

$$
\begin{gathered}
\eta_{s}=\frac{s-\hat{s}}{s}=\frac{\lambda_{0}-\lambda_{0}}{\lambda_{0}\left(\lambda_{0}-1\right)}=\frac{1-\hat{\lambda}}{\hat{\lambda}\left(\lambda_{0}-1\right)}=\frac{\eta_{\lambda_{0}}}{\hat{\lambda}\left(\lambda_{0}-1\right)}, \\
\eta_{s^{\prime}}=\frac{s^{\prime}-s^{\prime}}{s^{\prime}}=\frac{\frac{1}{\lambda_{0}}-\frac{1}{\lambda_{0}}}{\frac{1}{\lambda_{0}}}=1-\frac{1}{\hat{\lambda}}=\frac{\eta_{\lambda_{0}}}{\eta_{\lambda_{0}}-1},
\end{gathered}
$$

relative error between $s$ and $S$, ranged in absolute value from 3\% to $57.81 \%$ for Quality I (averaging $21.41 \%$ ), from $4.58 \%$ to $61.61 \%$ for Quality II (averaging $23.77 \%$ ), and from $4.12 \%$ to $62.25 \%$ for Quality III (averaging 23.69\%), always with $s \hat{S}$. Conversely, the relative error between $s^{\prime}$ and $S^{\prime}$, ranged in absolute value from $1.82 \%$ to $16.26 \%$ for Quality I (averaging $8.65 \%$ ), from $2.41 \%$ to $14.87 \%$ for Quality II (averaging $8.35 \%$ ), and from $1.85 \%$ to $12.62 \%$ for Quality III (averaging $7.03 \%$ ),

341 always with $s^{\prime}>S^{\prime}$. Therefore, in general it would be better to use $s^{\prime}$, rather than $\hat{S}$. Just as for $342 \eta_{\lambda_{0}}$ and $\eta_{\lambda}$, the maximum relative errors between $s$ and $\hat{S}, s^{\prime}$ and $\hat{S}^{\prime}$, occurred in any case for the $G=26 \mathrm{~m}^{2} /$ ha and $R=200$ stems $/$ ha scenarios, and the minimum for the $G=21 \mathrm{~m}^{2} /$ ha and $R=840$ stems/ha scenarios.

Additionally, we obtain the following results: 
346 1. As mentioned in the previous section, the variables $\lambda_{0}$ and $\lambda_{0}$ have a strong positive linear

347 correlation. Table 5 shows the regression parameters and Figure 1 depicts the points $\left(\lambda_{0}, \lambda_{0}\right)(198$

348 points for each Quality level) and the fitted linear regression line. In particular, the total proportion

349 of variance in $\lambda_{0}$ explained by $\lambda_{0}$ was $r^{2}=0.9961$ for Quality I, 0.9964 for Quality II, and 0.9966 for

350 Quality III.

351 2. The variables $s$ and $\hat{S}$ have a strong positive linear correlation (see Table 5). The total proportion of

352 variance in s explained by $\hat{S}$ was $r^{2}=0.9995$ for Quality I, 0.9993 for Quality II, and 0.9991 for Quality 353 III.

354 3. The variables $s^{\prime}$ and $S^{\prime}$ have a strong positive linear correlation, with the same $r^{2}$ values as 355 between $s$ and $\hat{S}($ see Table 5$)$.

356 4. By substituting the transition probabilities $p_{i}$ from Table 1 and the previously calculated $\lambda_{0}$ values 357 into (21), we obtained $W_{0}$, as summarized in Table 6 together with $W_{0}$ for some scenarios. We 358 evaluated the distance between $W_{0}$ and $W_{0}$ with Keyfitz's $\Delta$,

$$
\Delta\left(W_{0}, W_{0}\right)=\frac{1}{2} \sum_{i=1}^{n}\left|N_{i}-N_{i}\right|
$$

360 where $N_{i}$ and $N_{i}$ are the components of the diameter distributions $W_{0}$ and $W_{0}$, scaled to 1 (i.e.

$361 \sum_{i=1}^{n} N_{i}=\sum_{i=1}^{n} N_{i}=1$ ) (Keyfitz, 1968). The maximum value of $\Delta$ is 1 , and the minimum of 0 occurs

362 when the diameter distributions $W_{0}$ and $W_{0}$ (scaled to 1 ) are identical. Smaller values of $\Delta$ result

363 when the distributions $W_{0}$ and $W_{0}$ (scaled to 1 ) become more similar. As Table 6 shows, Keyfitz's $\Delta$ 
364 returns low values for all the scenarios and thus suggests that $W_{0}$ is a good approximation of $W_{0}$. The 365 minimum distance between $W_{0}$ and $W_{0}$ occurred in any case for the $G=21 \mathrm{~m}^{2} / \mathrm{ha}$ and $R=840$ 366 stems/ha scenarios, and the maximum for the $G=26 \mathrm{~m}^{2} /$ ha and $R=200$ stems $/$ ha scenarios.

\section{Discussion and Conclusion}

This study proposes a simple and direct method based on dimensionless numbers, obtained under conditions of stable equilibrium from a matrix model, to provide reliable approximations of the population growth rate, the "sustainable/stable" harvest rate, the proportion of trees that has to remain unharvested to retain the stable diameter distribution, and the stable diameter distribution, of a monospecific uneven-aged stand. Moreover, $\hat{s}$ and $s^{\prime}$ could also serve to estimate boundaries between sustainable and unsustainable harvesting. In fact, these dimensionless numbers are based on the following variables: (a) the transition probabilities $p_{k}$, which can be calculated from counts of observed transitions between adjacent diameter classes from $t$ to $t+1$. In the absence of a detailed census of the trees in the study area, they could be estimated using the methods proposed by López et al. (2007, 2008, 2012); (b) the basal area of the stand $G$, and the global amount of (natural) recruitment $R$ (defined as the total number of trees entering into the first diameter class from $t$ to $t+1$, in general above a certain threshold diameter). Although Pinus nigra regenerates well in the study area under its own canopy (Retana et al., 2002; Tiscar, 2007), there is a critical basal area to allow sufficient light transmittance for regeneration. This maximum value is around $20-30 \mathrm{~m}^{2} / \mathrm{ha}$ (Schütz, 1989; Serrada et al., 1994; Monge, 1997). On the other hand, basal areas clearly below 20 $\mathrm{m}^{2} /$ ha (before harvesting) could compromise natural regeneration (Serrada et al., 1994). Consequently, we have considered, in addition to three levels of diameter growth (Qualities I-III), 6 levels of stand basal area from $G=21$ to $G=26 \mathrm{~m}^{2} / \mathrm{ha}$, and 33 levels of global recruitment, from $R=$ 
388 planning scenarios. The corresponding problems simulate a wide variety of typical management 389 cases in the study area; and (c) the number $n$ and width $w$ of the diameter classes.

Disregarding those scaling factors $n$ and $w$ and the constant terms, the core of these dimensionless numbers is given by the quotient

$$
\gamma_{G}=\frac{R \prod_{i=1}^{n-1} p_{i}}{G}
$$

393 which represents the product of the stand growth inputs, $R$ and $\prod_{i=1}^{n-1} p_{i}$, per unit of basal area (since $394 V_{G}$ is measured in stem $/ \mathrm{m}^{2}, \gamma_{G}$ has dimension $L^{-2}$ ). In this regard, the higher the level of recruitment and the faster the flow of the stems through the diameter classes, for a given basal area, the greater the population growth rate. Furthermore, there is also a strong positive linear correlation between

$397 \lambda_{0}$ and $\gamma_{G}$, though a little bit weaker than that seen between $\lambda_{0}$ and $\lambda_{0}$, with $r^{2}$ being 0.9874 for 398 Quality I, 0.9876 for Quality II, and 0.9879 for Quality III. This suggests that the scaling factors $n$ and 399 $w$ could have limited influence on $\lambda_{0}$ and $\lambda_{0}$.

As shown in Section 3, the relative errors between $\lambda_{0}$ and $\lambda_{0}, \lambda$ and $\hat{\lambda}, s$ and $\hat{S}, s^{\prime}$ and $S^{\prime}$, in general were small, in particular for Quality III scenarios, with the lowest basal area and the highest recruitment scenarios providing the most accurate approximations. This result is even more noteworthy given the fact that possibly the most likely scenarios would be those with basal areas between $21-22 \mathrm{~m}^{2} / \mathrm{ha}$ and abundant recruitment, especially bearing in mind that the highest population growth rates $\lambda_{0}$ (and, by extension, "sustainable/stable" harvest rates $s$ ) were obtained for the lowest basal area and the highest recruitment scenarios.

Those small relative errors between $\lambda_{0}$ and $\lambda_{0}$ are not due to partial or total self-cancellation of the 
409 attributable to the fact that the factor $a_{n}=\frac{16 G}{\pi w^{2} R}$ is present in all the coefficients of $P_{n}\left(\lambda_{0}\right)$ on the 410 one hand, and the impact of the products of successive transition probabilities on those coefficients 411 (which, accumulating more transition probabilities at each product as the order of the coefficient 412 diminishes, show a decreasing influence) on the other. Hence the scenarios with the lowest relative 413 errors were those corresponding to Quality III (where the transition probabilities are lowest), with 414 low basal area and high recruitment values enhancing those effects. In worst-case scenarios (Quality 415 I, high basal area and low recruitment), the coefficient $a_{n-1}$ would also have some influence on $\lambda_{0}$, 416 although less than $a_{n}$. In either case, as depicted in Figure $2, P_{n}\left(\lambda_{0}\right)$ provides a good approximation to 417 obtain the dominant root of $P_{n}\left(\lambda_{0}\right)$ in the vicinity of $\lambda_{0}=1$, especially in the lowest basal area and the 418 highest recruitment scenarios. Conversely, the convergence of the successive approximations to the 419 root greater than 1 given by the linear (or tangent line) approximation of $P_{n}\left(\lambda_{0}\right)$, is very slow if we 420 start the process with $\lambda_{0}=1$, due to the shape of the graph of $P_{n}\left(\lambda_{0}\right)$ in the vicinity of $\lambda_{0}=1$.

421 Furthermore, the strong positive linear correlation between $\lambda_{0}$ and $\lambda_{0}$ means (among other things) 422 that stratifying the forest into uniform areas based upon tree diameter growth, the same linear 423 regression line can be used in each stratum to estimate the population growth rate (this also applies 424 to $s$ and $s^{\prime}$ ). Moreover, only two homogeneous sample plots are required to obtain the parameters 425 of those linear regressions for each quality site.

As shown in the Introduction, population growth rates from matrix models are calculated to assess, over the long-term, whether a population is expected to grow, remain stable or decline. With this broader view, the population growth rate and the asymptotic stock recovery rate that is used in tropical forests to assess logging sustainability (Picard et al., 2009) (dimensionless quantities), 430 probably also might be considered dimensionless numbers, but there are not simple and direct 431 expressions relating them to the inputs of the problem. In this regard, this study showsa simple and 432 direct method based on dimensionless numbers to provide reliable approximations $\phi_{0}, \hat{S}_{,} \bar{S}^{\prime}$ and 
$433 W_{0}$ of the population growth rate $\lambda_{0}$, the "sustainable/stable" harvest rate $s$, the proportion of trees 434 that has to remain unharvested to retain the stable diameter distribution $s^{\prime}$, and the stable diameter 435 distribution $W_{0}$. Moreover, $\hat{S}$ and $S^{\prime}$ could also serve to estimate boundaries between sustainable 436 and unsustainable harvesting. The best approximations occurred in any case for the lowest level of 437 diameter growth, the lowest basal area, and the highest recruitment scenarios. Furthermore, the 438 study reveals the existence of a strong positive linear correlation across different stand attributes 439 between $\lambda_{0}$ and $\lambda_{0}, s$ and $\hat{S}, s^{\prime}$ and $S^{\prime}$, as well as a small distance between the stable diameter 440 distribution of the stand $W_{0}$ and its approximation $W_{0}$.

442 Conflict of interest statement

$443 \quad$ None.

\section{References}

445 Buongiorno, J. and Michie, B.R., 1980. A matrix model of uneven-aged forest management. For Sci 446 26:609-625.

Caswell, H., 1978. A general formula for the sensitivity of population growth rate to changes in life history parameters. Theoretical Population Biology 14:215-230.

Caswell, H., 2001. Matrix Population Models: Construction, Analysis, and Interpretation, 2nd ed.; Sinauer Associates Inc.: Sunderland, MA, USA, 2001; p. 713.

Gotelli, N.J., 2001. A Primer of Ecology, 3rd edn. Sinauer Associates, Sunderland, MA.

Grande, M.A. and García Abril, A., 2005. Los Pinares de Pinus nigra Arn. en España: Ecología, uso y gestión; Fundación Conde del Valle de Salazar (FUCOVASA): Madrid, Spain, 2005; pp. 1-53.

Hirsch, M., and Smale, S., 1974. Differential Equations, Dynamical Systems, and Linear Algebra. Academic Press Inc. NY 10003, USA.

Keyfitz, N., 1968. Introduction to the mathematics of population. Addison-Wesley, Reading, MA.

Liang, J., Buongiorno, J., and Monserud, R.A., 2005. Growth and Yield of All-aged Douglas-fir/western hemlock Stands: A Matrix Model with Stand Diversity Effects. Canadian Journal of Forest Research 465 35: 2369-2382. 
466 Liang, J. and Picard, N., 2012. Matrix Model of Forest Dynamics: An Overview and Outlook. Forest

467 Science 59(3): 359-378.

468

López, I., Ortuño, S.F., Martín, A.J. and Fullana, C. 2007 Estimating the sustainable harvesting and the stable diameter distribution of European beech with projection matrix models. Ann. For. Sci. 64: 593-599.

López, I., Fullana, C., Ortuño, S.F. and Martín, A.J., 2008. Choosing Fagus sylvatica L. matrix model dimension by sensitivity analysis of the population growth rate with respect to the width of the diameter classes. Ecological Modelling 218: 307-314.

López, I., Ortuño, S., García, F. and Fullana, C., 2012. Is De Liocourt's distribution stable? Forest Science Volume 58, Number 1, February 2012, 34-46.

López, I., Ortuño, S., García, F. and Fullana, C., 2013. Are the economically optimal harvesting strategies of uneven-aged Pinus nigra stands always sustainable and stabilizing? Forests 2013, 4, 830-848.

Maple, Version 18.0; Maplesoft: Ontario, Canada, 2014.

Monge Reyes, A., 1997. Selvicultura y regeneración de Pinus nigra en las sierras Béticas. Doctoral Thesis. ETSI de Montes de la UPM. Spain.

Picard, N., Ngok Banak, L., Namkosserena, S., and Yalibanda, Y., 2009. The stock recovery rate in a Central African rain forest: an index of sustainability based on projection matrix models. Canadian Journal of Forest Research 39(11): 2138-2152.

Retana, J., Espelta, J.M., Habrouk, A., Ordóñez, J.L., and Solà-Morales, F., 2002. Regeneration patterns of three Mediterranean pines and forest changes after a large wildfire in North-eastern Spain. Ecoscience 9:89-97.

Schütz, J-P., 1989. Le régime du Jardinage. E.T.H. Zürich, Switzerland.

Schütz, J.P., 2006. Modelling the demographic sustainability of pure beech plenter forests in Eastern Germany. Ann. For. Sci., 63, 93-100.

Serrada, R., Domínguez Lerena, S., Sánchez Resco, M.I., and Ruiz Ortiz, J., 1994. El problema de la regeneración natural de Pinus nigra Arn. Revista Montes 36, Madrid, Spain.

Szirtes, T., 2007. Applied Dimensional Analysis and Modeling, Second Edition. Elsevier ButterworthHeinemann, 820p.

Tiscar, P.A., 2007. Dinámica de regeneración de Pinus nigra Subs. Salzmannii al sur de su área de distribución: etapas, procesos y factores implicados. Investigación Agraria: Sistemas y Recursos Forestales 16(2): 124-135. 
511 Vanclay, J. K., 2012. Modelling continuous cover forests. In Continuous Cover Forestry (pp. 229-241).

512 Springer Netherlands.

513

514

515

516

517

518

519

520

521

522

523

524

525

526

527

528

529

530
Zhou, M. and J. Buongiorno, J., 2004. Nonlinearity and Noise Interaction in a Model of Forest Growth. Ecological Modelling 180: 291-304.

\section{Table and Figure captions}

Figure 1: Linear regression between $\lambda_{0}$ and $\lambda_{0}$ (left: Quality I; middle: Quality II; right: Quality III).

Figure 2: Graph of the polynomials $P_{n}\left(\lambda_{0}\right)$ (introduced in equation (10)) and $P_{n}\left(\lambda_{0}\right)$ (as defined in equation (13)) (left: Quality I; middle: Quality II; right: Quality III; first row: $G=21 \mathrm{~m}^{2} /$ ha and $R=840$ stem/ha scenarios; second row: $G=26 \mathrm{~m}^{2} /$ ha and $R=200$ stem/ha scenarios).

Table 1: Transition probabilities between diameter classes for each quality ( $n=$ number of diameter classes; $w=$ width of the diameter classes $=0.06$ meters ).

Table 2: Numerical values of $\lambda_{0}$ (as defined in equation (10)), $s$ (as defined in equation (3)), $s^{\prime}$ (with $s^{\prime}=1-s$ ), $\hat{\lambda}_{0}$ (as defined in equation (15)), $\hat{\lambda}$ (as defined in equation (16)), $s^{\prime}$ (as defined in equation (20)) and $\hat{s}$ (as defined in equation (19)), for Quality I.

Table 3: Numerical values of $\lambda_{0}$ (as defined in equation (10)), $s$ (as defined in equation (3)), $s^{\prime}$ (with $s^{\prime}=1-s$ ), $\hat{\lambda}_{0}$ (as defined in equation (15)), $\hat{\lambda}$ (as defined in equation (16)), $s^{\prime}$ (as defined in equation (20)) and $\hat{s}$ (as defined in equation (19)), for Quality II.

Table 4: Numerical values of $\lambda_{0}$ (as defined in equation (10)), $s$ (as defined in equation (3)), $s^{\prime}$ (with $s^{\prime}=1-s$ ), $\hat{\lambda}_{0}$ (as defined in equation (15)), $\hat{\lambda}$ (as defined in equation (16)), $s^{\prime}$ (as defined in equation (20)) and $\hat{s}$ (as defined in equation (19)), for Quality III.

Table 5: Regressions between: $\lambda_{0}$ (as defined in equation (10)) and $\hat{\lambda}_{0}$ (as defined in equation (15)); $s$ (as defined in equation (3)) and $\hat{s}$ (as defined in equation (19)); $s^{\prime}$ (with $s^{\prime}=1-s$ ) and $s^{\prime}$ (as defined in equation (20)) ( $r^{2}$ = coefficient of determination; $r_{a d j}^{2}$ = adjusted coefficient of determination; Linear regressions: $a=$ slope with its associated 95\% confidence interval, $b=$ intercept with its associated $95 \%$ confidence interval; Number of points $=198$ in each case).

Table 6: Stable diameter distributions $\left(W_{0}\right)$ and approximation $\left(W_{0}\right)$ (as defined in equation (21)), scaled to 1, along with Keyfitz's $\Delta$. 
TABLES

Table 1: Transition probabilities between diameter classes for each quality $(n=$ number of diameter classes; $w=$ width of 546 the diameter classes $=0.06$ meters).

\begin{tabular}{|c|c|c|c|}
\hline & Quality I & Quality II & Quality III \\
\hline$n$ & 9 & 8 & 7 \\
\hline$(0,6] \rightarrow(6,12]$ & $p_{1}=0.7697$ & $p_{1}=0.5951$ & $p_{1}=0.4564$ \\
\hline$(6,12] \rightarrow(12,18]$ & $p_{2}=0.8602$ & $p_{2}=0.6824$ & $p_{2}=0.5326$ \\
\hline$(12,18] \rightarrow(18,24]$ & $p_{3}=0.7913$ & $p_{3}=0.6200$ & $p_{3}=0.4697$ \\
\hline$(18,24] \rightarrow(24,30]$ & $p_{4}=0.6828$ & $p_{4}=0.5190$ & $p_{4}=0.3692$ \\
\hline$(24,30] \rightarrow(30,36]$ & $p_{5}=0.5533$ & $p_{5}=0.3971$ & $p_{5}=0.2475$ \\
\hline$(30,36] \rightarrow(36,42]$ & $p_{6}=0.4106$ & $p_{6}=0.2618$ & $p_{6}=0.1119$ \\
\hline$(36,42] \rightarrow(42,48]$ & $p_{7}=0.2587$ & $p_{7}=0.1171$ & - \\
\hline$(42,48] \rightarrow(48, \rightarrow)$ & $p_{8}=0.1000$ & - & - \\
\hline$\prod_{i=1}^{n-1} p_{i}$ & 0.002104 & 0.001590 & 0.001168 \\
\hline
\end{tabular}

547

548

549

550

551

552

553

554

555

556

557

558

559

560

561 
562 Table 2: Numerical values of $\lambda_{0}$ (as defined in equation (10)), $s$ (as defined in equation (3)), $s^{\prime}$ (with $s^{\prime}=1-s$ ), $\hat{\lambda}_{0}$ (as defined in equation (15)), $\hat{\lambda}$ (as defined in equation (16)), $s^{\prime}$ (as defined in equation (20)) and $\hat{s}$ (as defined in equation (19)), for Quality I.

\begin{tabular}{|c|c|c|c|c|}
\hline & & $R=200$ stem $/$ ha & $R=520$ stem $/$ ha & $R=840$ stem $/$ ha \\
\hline \multirow{7}{*}{$\begin{array}{l}G=22 \\
\mathrm{~m}^{2} / \mathrm{ha}\end{array}$} & $\lambda_{0}$ & 1.305091 & 1.477671 & 1.594240 \\
\hline & $s$ & 0.233770 & 0.323259 & 0.372742 \\
\hline & $s^{\prime}=\lambda_{0}^{-1}$ & 0.766230 & 0.676741 & 0.627258 \\
\hline & $\lambda_{0}$ & 1.540047 & 1.600537 & 1.633405 \\
\hline & $\widehat{\lambda}$ & 1.180031 & 1.083148 & 1.024566 \\
\hline & $s^{\prime}$ & 0.649331 & 0.624790 & 0.612218 \\
\hline & $\hat{s}$ & 0.350669 & 0.375210 & 0.387782 \\
\hline \multirow{7}{*}{$\begin{array}{l}G=24 \\
\mathrm{~m}^{2} / \mathrm{ha}\end{array}$} & $\lambda_{0}$ & 1.292499 & 1.458959 & 1.571264 \\
\hline & $s$ & 0.226305 & 0.314580 & 0.363570 \\
\hline & $s^{\prime}=\lambda_{0}^{-1}$ & 0.773695 & 0.685420 & 0.636430 \\
\hline & $\lambda_{0}$ & 1.534851 & 1.594759 & 1.627310 \\
\hline & $\hat{\lambda}$ & 1.187507 & 1.093080 & 1.035669 \\
\hline & $S^{\prime}$ & 0.651529 & 0.627054 & 0.614511 \\
\hline & $\hat{S}$ & 0.348471 & 0.372946 & 0.385489 \\
\hline \multirow{7}{*}{$\begin{array}{l}G=26 \\
\mathrm{~m}^{2} / \mathrm{ha}\end{array}$} & $\lambda_{0}$ & 1.281305 & 1.442342 & 1.550881 \\
\hline & $s$ & 0.219546 & 0.306683 & 0.355205 \\
\hline & $s^{\prime}=\lambda_{0}^{-1}$ & 0.780454 & 0.693317 & 0.644795 \\
\hline & $x_{0}$ & 1.530115 & 1.589493 & 1.621756 \\
\hline & $\hat{\lambda}$ & 1.194185 & 1.102022 & 1.045700 \\
\hline & $s^{\prime}$ & 0.653546 & 0.629132 & 0.616616 \\
\hline & $\hat{S}$ & 0.346454 & 0.370868 & 0.383384 \\
\hline
\end{tabular}


565 Table 3: Numerical values of $\lambda_{0}$ (as defined in equation (10)), $s$ (as defined in equation (3)), $s^{\prime}$ (with $s^{\prime}=1-s$ ), $\hat{\lambda}_{0}$ (as 566 567 defined in equation (15)), $\hat{\lambda}$ (as defined in equation (16)), $s^{\prime}$ (as defined in equation (20)) and $\hat{s}$ (as defined in equation (19)), for Quality II.

\begin{tabular}{|c|c|c|c|c|}
\hline & & $R=200$ stem $/$ ha & $R=520$ stem $/$ ha & $R=840$ stem $/$ ha \\
\hline \multirow{7}{*}{$\begin{array}{l}G=22 \\
\mathrm{~m}^{2} / \mathrm{ha}\end{array}$} & $\lambda_{0}$ & 1.262093 & 1.412527 & 1.514472 \\
\hline & $s$ & 0.207665 & 0.292049 & 0.339704 \\
\hline & $s^{\prime}=\lambda_{0}^{-1}$ & 0.792335 & 0.707951 & 0.660296 \\
\hline & $\lambda_{0}$ & 1.467953 & 1.527320 & 1.559897 \\
\hline & $\hat{\lambda}$ & 1.163111 & 1.081267 & 1.029994 \\
\hline & $s^{\prime}$ & 0.681221 & 0.654742 & 0.641068 \\
\hline & $\hat{s}$ & 0.318779 & 0.345258 & 0.358932 \\
\hline \multirow{7}{*}{$\begin{array}{l}G=24 \\
\mathrm{~m}^{2} / \mathrm{ha}\end{array}$} & $\lambda_{0}$ & 1.251140 & 1.396188 & 1.494358 \\
\hline & $s$ & 0.200729 & 0.283764 & 0.330816 \\
\hline & $s^{\prime}=\lambda_{0}^{-1}$ & 0.799271 & 0.716236 & 0.669184 \\
\hline & $\lambda_{0}$ & 1.462891 & 1.521615 & 1.553841 \\
\hline & $\hat{\lambda}$ & 1.169247 & 1.089835 & 1.039805 \\
\hline & $s^{\prime}$ & 0.683578 & 0.657196 & 0.643567 \\
\hline & $\hat{S}$ & 0.316422 & 0.342804 & 0.356433 \\
\hline \multirow{7}{*}{$\begin{array}{l}G=26 \\
\mathrm{~m}^{2} / \mathrm{ha}\end{array}$} & $\lambda_{0}$ & 1.241406 & 1.381684 & 1.476521 \\
\hline & $s$ & 0.194462 & 0.276245 & 0.322732 \\
\hline & $s^{\prime}=\lambda_{0}^{-1}$ & 0.805538 & 0.723755 & 0.677268 \\
\hline & $x_{0}$ & 1.458283 & 1.516422 & 1.548327 \\
\hline & $\hat{\lambda}$ & 1.174702 & 1.097518 & 1.048632 \\
\hline & $s^{\prime}$ & 0.685738 & 0.659447 & 0.645858 \\
\hline & $\hat{S}$ & 0.314262 & 0.340553 & 0.354142 \\
\hline
\end{tabular}


Table 4: Numerical values of $\lambda_{0}$ (as defined in equation (10)), $s$ (as defined in equation (3)), $s^{\prime}$ (with $s^{\prime}=1-s$ ), $\hat{\lambda}_{0}$ (as defined in equation (15)), $\hat{\lambda}$ (as defined in equation (16)), $s^{\prime}$ (as defined in equation (20)) and $\hat{s}$ (as defined in equation (19)), for Quality III.

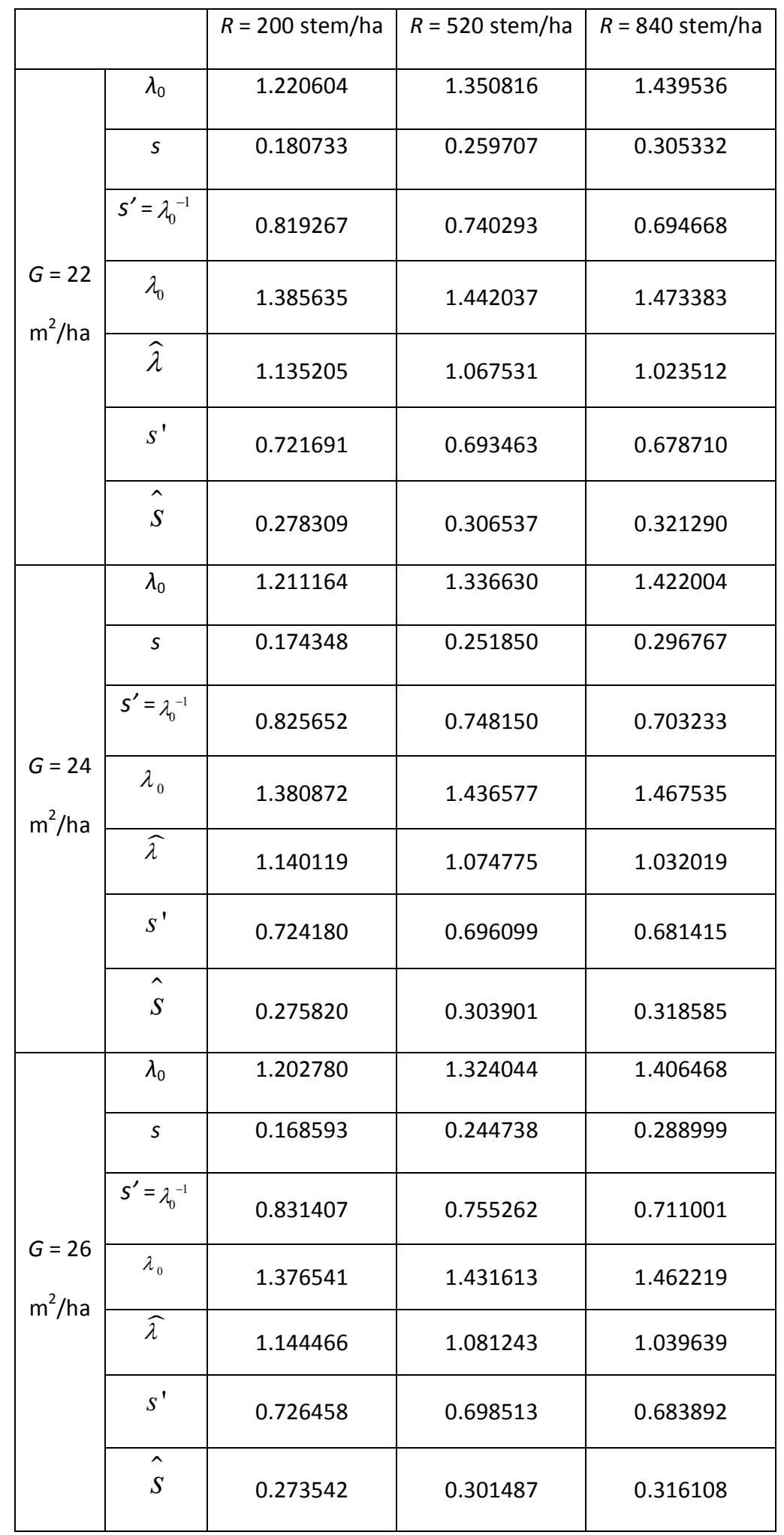


571

572

573

574

Table 5: Regressions between: $\lambda_{0}$ (as defined in equation (10)) and $\hat{\lambda}_{0}$ (as defined in equation (15)); $s$ (as defined in equation (3)) and $\hat{S}$ (as defined in equation (19)); $s^{\prime}$ (with $\left.s^{\prime}=1-s\right)$ and $s^{\prime}\left(\right.$ as defined in equation (20)) $\left(r^{2}=\right.$ coefficient of determination; $r^{2}{ }_{a d j}=$ adjusted coefficient of determination; Linear regressions: $a=$ slope with its associated $95 \%$ confidence interval, $b=$ intercept with its associated $95 \%$ confidence interval; Number of points $=198$ in each case).

\begin{tabular}{|c|c|c|c|c|c|}
\hline & & $r^{2}$ & $r_{a d j}^{2}$ & $a$ & $b$ \\
\hline \multirow{3}{*}{ QI } & $\begin{array}{l}\text { regression } \\
\text { of } \lambda_{0} \text { on } \lambda_{0}\end{array}$ & 0.9961 & 0.9961 & $\begin{array}{c}3.0823 \\
(3.0552,3.1093)\end{array}$ & $\begin{array}{c}-3.4514 \\
(-3.4944,-3.4084)\end{array}$ \\
\hline & $\begin{array}{c}\text { regression } \\
\text { of } s \text { on } \hat{S}\end{array}$ & 0.9995 & 0.9995 & $\begin{array}{c}3.7471 \\
(3.7350,3.7593)\end{array}$ & $\begin{array}{c}-1.0821 \\
(-1.0866,-1.0776)\end{array}$ \\
\hline & $\begin{array}{l}\text { regression } \\
\text { of } s^{\prime} \text { on } s^{\prime}\end{array}$ & 0.9995 & 0.9995 & $\begin{array}{c}3.7471 \\
(3.7350,3.7593)\end{array}$ & $\begin{array}{c}-1.6651 \\
(-1.6727,-1.6575)\end{array}$ \\
\hline \multirow{3}{*}{ Q॥ } & $\begin{array}{l}\text { regression } \\
\text { of } \lambda_{0} \text { on } \lambda_{0}\end{array}$ & 0.9964 & 0.9964 & $\begin{array}{c}2.7319 \\
(2.7087,2.7551)\end{array}$ & $\begin{array}{c}-2.7564 \\
(-2.7917,-2.7211)\end{array}$ \\
\hline & $\begin{array}{c}\text { regression } \\
\text { of } s \text { on } \hat{S}\end{array}$ & 0.9993 & 0.9993 & $\begin{array}{c}3.2894 \\
(3.2771,3.3018)\end{array}$ & $\begin{array}{c}-0.8429 \\
(-0.8471,-0.8387)\end{array}$ \\
\hline & $\begin{array}{l}\text { regression } \\
\text { of } s^{\prime} \text { on } s^{\prime}\end{array}$ & 0.9993 & 0.9993 & $\begin{array}{c}3.2894 \\
(3.2771,3.3018)\end{array}$ & $\begin{array}{c}-1.4465 \\
(-1.4546,-1.4384)\end{array}$ \\
\hline \multirow{3}{*}{ Q III } & $\begin{array}{l}\text { regression } \\
\text { of } \lambda_{0} \text { on } \lambda_{0}\end{array}$ & 0.9966 & 0.9966 & $\begin{array}{c}2.4835 \\
(2.4631,2.5039)\end{array}$ & $\begin{array}{c}-2.2275 \\
(-2.2567,-2.1983)\end{array}$ \\
\hline & $\begin{array}{c}\text { regression } \\
\hat{\text { of } s \text { on } S}\end{array}$ & 0.9991 & 0.9991 & $\begin{array}{c}2.8989 \\
(2.8868,2.9110)\end{array}$ & $\begin{array}{c}-0.6281 \\
(-0.6318,-0.6245)\end{array}$ \\
\hline & $\begin{array}{l}\text { regression } \\
\text { of } s^{\prime} \text { on } s^{\prime}\end{array}$ & 0.9991 & 0.9991 & $\begin{array}{c}2.8989 \\
(2.8868,2.9110)\end{array}$ & $\begin{array}{c}-1.2708 \\
(-1.2792,-1.2623)\end{array}$ \\
\hline
\end{tabular}

575

576

577

578

579

580

581

582

583

584

585 
Table 6: Stable diameter distributions $\left(W_{0}\right)$ and approximation $\left(W_{0}\right)$ (as defined in equation (21)), scaled to 1 , along with Keyfitz's $\Delta$.

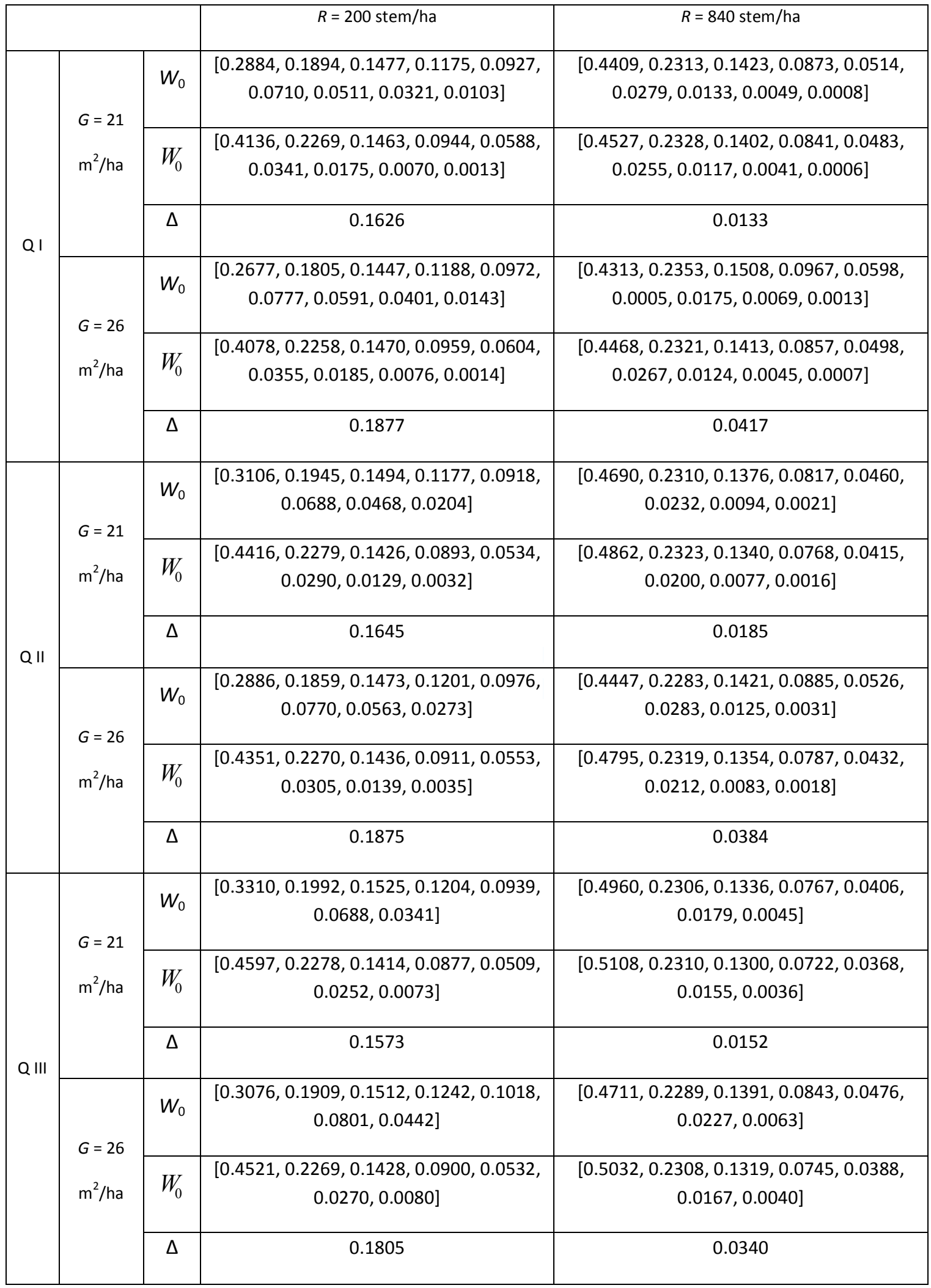


FIGURES

Figure 1: Linear regression between $\lambda_{0}$ and $\lambda_{0}$ (left: Quality I; middle: Quality II; right: Quality III).
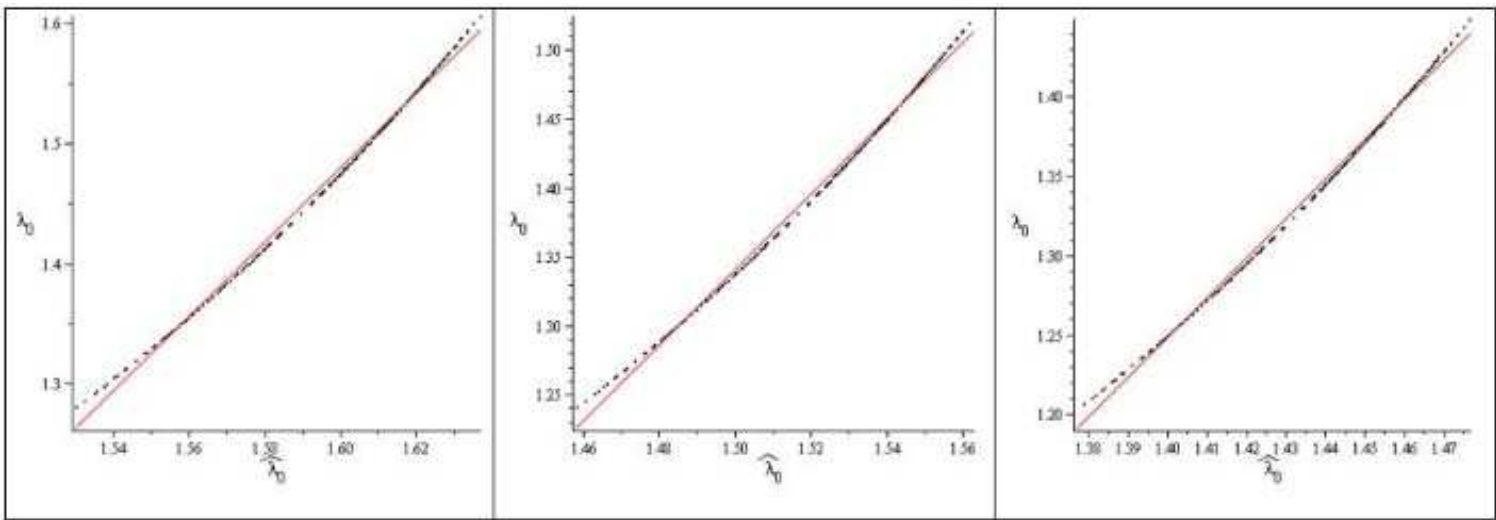

592

593

594

595

596

597

598

599

600

601

602

603

604

605

606

607

608 
Figure 2: Graph of the polynomials $P_{n}\left(\lambda_{0}\right)$ (introduced in equation (10)) and $P_{n}\left(\lambda_{0}\right)$ (as defined in equation (13)) (left: Quality I; middle: Quality II; right: Quality III; first row: $G=21 \mathrm{~m}^{2} /$ ha and $R=840$ stem/ha scenarios; second row: $G=26$ $\mathrm{m}^{2} /$ ha and $R=200$ stem/ha scenarios).

609

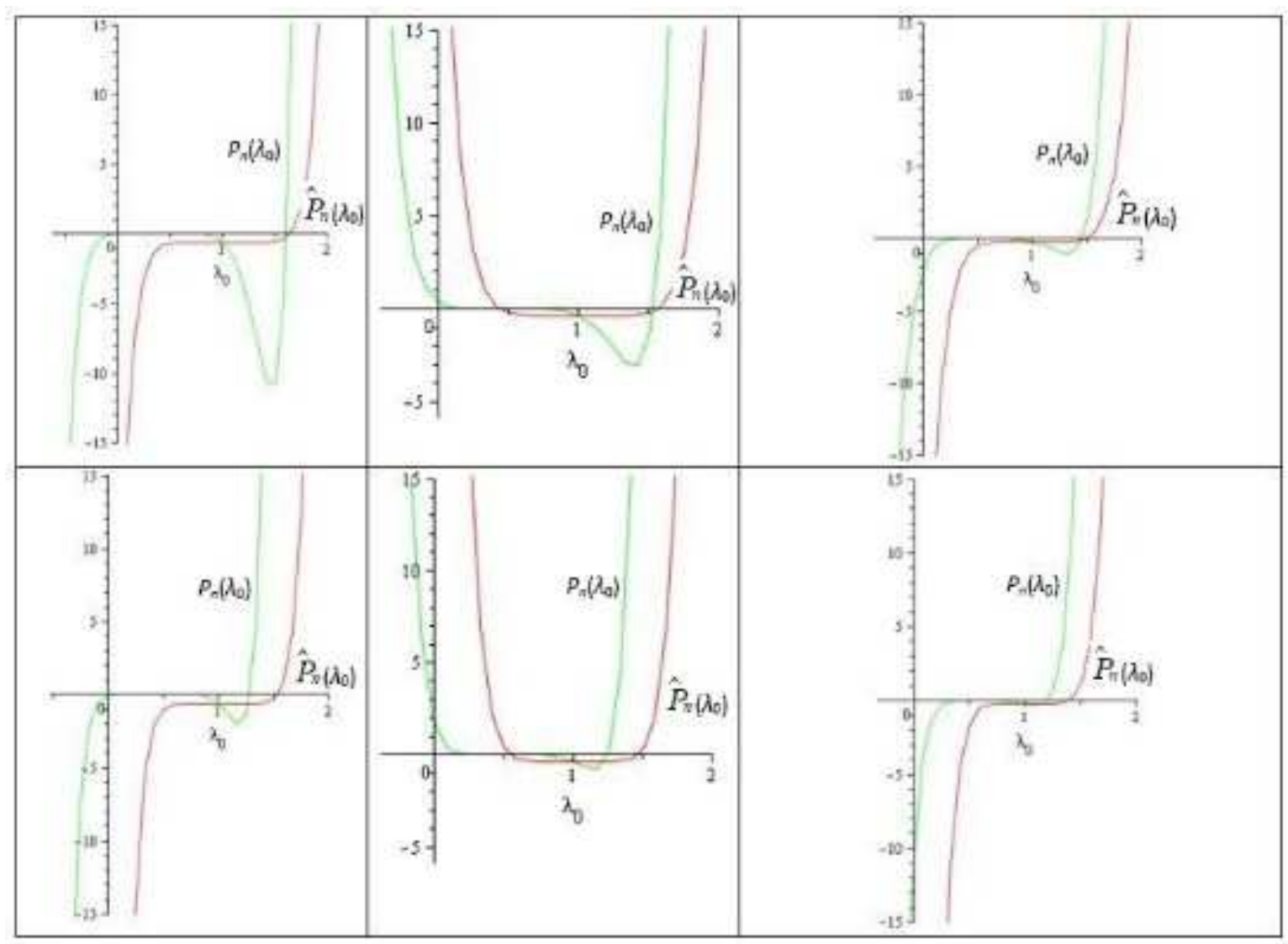

610 
$622 a_{n}=\frac{16 G}{\pi w^{2} R}$.

$a_{n-1}=\frac{16 G}{\pi w^{2} R}\left(\sum_{i=1}^{n-1} p_{i}\right)-1^{2}$.

624

$a_{n-2}=\frac{16 G}{\pi w^{2} R}\left(\begin{array}{c}\sum_{i=1}^{n-2} p_{i} p_{j} \\ (i<j)\end{array}\right)-1^{2}\left(\sum_{i=2}^{n-1} p_{i}\right)-3^{2} p_{1}$

625

$a_{n-3}=\frac{16 G}{\pi w^{2} R}\left(\begin{array}{l}n-3 \\ i=1 \\ (i<j<k)\end{array}\right)-1_{i}^{2}\left(\sum_{i=2}^{n-2} p_{i} p_{j}\right)-3^{2} p_{1} \sum_{i=3}^{n-1} p_{i}-5^{2} p_{1} p_{2}$

626

$a_{n-4}=\frac{16 G}{\pi w^{2} R}\left(\sum_{i=1}^{n-4} p_{i} p_{j} p_{k} p_{l}\right)-1^{2}\left(\sum_{i=2}^{n-3} p_{i} p_{j} p_{k}\right)-3^{2} p_{1}\left(\sum_{i=3}^{n-2} p_{i} p_{j}\right)-5^{2} p_{1} p_{2} \sum_{i=4}^{n-1} p_{i}-7^{2} p_{1} p_{2} p_{3}$.

627

628

$a_{1}=a_{n-(n-1)}=\frac{16 G}{\pi w^{2} R} \prod_{i=1}^{n-1} p_{i}-1^{2} \prod_{i=2}^{n-1} p_{i}-3^{2} p_{1} \prod_{i=3}^{n-1} p_{i}-5^{2} p_{1} p_{2} \prod_{i=4}^{n-1} p_{i}-7^{2} p_{1} p_{2} p_{3} \prod_{i=5}^{n-1} p_{i}-$

$-9^{2} p_{1} p_{2} p_{3} p_{4} \prod_{i=6}^{n-1} p_{i}-11^{2} p_{1} p_{2} p_{3} p_{4} p_{5} \prod_{i=7}^{n-1} p_{i}-13^{2} p_{1} p_{2} p_{3} p_{4} p_{5} p_{6} \prod_{i=8}^{n-1} p_{i}-\ldots-(2 n-3)^{2} \prod_{i=1}^{n-2} p_{i}$

629 with $\prod_{i=a}^{b} p_{i}=0$ if $a>b$

630 (note that $\prod_{i=1}^{n-1} p_{i}=\sum_{\substack{i_{1}=1 \\\left(i_{1}<i_{2}<i_{3}<\ldots<i_{n-1}\right)}}^{n-(n-1)} p_{i_{1}} p_{i_{2}} p_{i_{3}} \ldots p_{i_{n-1}}, \prod_{i=2}^{n-1} p_{i}=\sum_{\substack{i_{2}=2 \\\left(i_{2}<i_{3}<i_{4}<\ldots<i_{n-1}\right)}}^{n-(n-2)} p_{i_{2}} p_{i_{3}} p_{i_{4}} \ldots p_{i_{n-1}}$, and so on).

631

$$
a_{0}=a_{n-n}=-(2 n-1)^{2} \prod_{i=1}^{n-1} p_{i}
$$


634

635

636

637

638

639

641

642

640

\section{Appendix 2: Coefficients of the polynomial $P_{n}\left(\lambda_{0}\right)=a_{n}\left(\lambda_{0}-1\right)^{n}+\ldots+a_{1}\left(\lambda_{0}-1\right)+a_{0}$ for $n=7$.}

$a_{7}=\frac{16 G}{\pi w^{2} R}$.

$a_{6}=\frac{16 G}{\pi w^{2} R}\left(p_{1}+p_{2}+p_{3}+p_{4}+p_{5}+p_{6}\right)-1$.

$a_{5}=\frac{16 G}{\pi w^{2} R}\left(p_{4} p_{5}+p_{3} p_{5}+p_{3} p_{4}+p_{5} p_{6}+p_{3} p_{6}+p_{2} p_{5}+p_{2} p_{3}+p_{4} p_{6}+p_{2} p_{6}+p_{2} p_{4}+\right.$

$\left.+p_{1} p_{6}+p_{1} p_{5}+p_{1} p_{4}+p_{1} p_{3}+p_{1} p_{2}\right)-p_{2}-p_{3}-p_{4}-p_{5}-p_{6}-9 p_{1}$.

$a_{4}=\frac{16 G}{\pi w^{2} R}\left(p_{2} p_{5} p_{6}+p_{4} p_{5} p_{6}+p_{1} p_{5} p_{6}+p_{2} p_{3} p_{6}+p_{3} p_{4} p_{5}+p_{3} p_{4} p_{6}+p_{2} p_{4} p_{5}+p_{2} p_{4} p_{6}+\right.$

$+p_{2} p_{3} p_{5}+p_{1} p_{2} p_{5}+p_{1} p_{2} p_{6}+p_{1} p_{3} p_{4}+p_{1} p_{3} p_{5}+p_{1} p_{3} p_{6}+p_{1} p_{4} p_{5}+p_{1} p_{4} p_{6}+p_{1} p_{2} p_{3}+$

$\left.+p_{1} p_{2} p_{4}+p_{3} p_{5} p_{6}+p_{2} p_{3} p_{4}\right)-p_{2} p_{3}-p_{3} p_{4}-p_{2} p_{4}-p_{2} p_{5}-p_{3} p_{5}-p_{4} p_{5}-p_{2} p_{6}-p_{3} p_{6}-$

$-p_{4} p_{6}-p_{5} p_{6}-9\left(p_{1} p_{3}+p_{1} p_{4}+p_{1} p_{5}+p_{1} p_{6}\right)-25 p_{1} p_{2}$.

$a_{3}=\frac{16 G}{\pi w^{2} R}\left(p_{1} p_{4} p_{5} p_{6}+p_{1} p_{3} p_{4} p_{5}+p_{2} p_{3} p_{5} p_{6}+p_{1} p_{2} p_{3} p_{5}+p_{1} p_{3} p_{4} p_{6}+p_{1} p_{2} p_{3} p_{6}+\right.$

$+p_{1} p_{2} p_{3} p_{4}+p_{2} p_{4} p_{5} p_{6}+p_{1} p_{2} p_{5} p_{6}+p_{1} p_{3} p_{5} p_{6}+p_{1} p_{2} p_{4} p_{5}+p_{1} p_{2} p_{4} p_{6}+p_{2} p_{3} p_{4} p_{6}+$

$\left.+p_{3} p_{4} p_{5} p_{6}+p_{2} p_{3} p_{4} p_{5}\right)-p_{2} p_{3} p_{5}-p_{2} p_{4} p_{5}-p_{2} p_{3} p_{4}-p_{3} p_{4} p_{5}-p_{2} p_{3} p_{6}-p_{3} p_{4} p_{6}-$

$-p_{2} p_{4} p_{6}-p_{2} p_{5} p_{6}-p_{3} p_{5} p_{6}-p_{4} p_{5} p_{6}-9\left(p_{1} p_{3} p_{4}+p_{1} p_{3} p_{5}+p_{1} p_{4} p_{5}+p_{1} p_{3} p_{6}+\right.$

$\left.+p_{1} p_{4} p_{6}+p_{1} p_{5} p_{6}\right)-25\left(p_{1} p_{2} p_{4}+p_{1} p_{2} p_{5}+p_{1} p_{2} p_{6}\right)-49 p_{1} p_{2} p_{3}$.

$a_{2}=\frac{16 G}{\pi w^{2} R}\left(p_{2} p_{3} p_{4} p_{5} p_{6}+p_{1} p_{2} p_{4} p_{5} p_{6}+p_{1} p_{2} p_{3} p_{5} p_{6}+p_{1} p_{2} p_{3} p_{4} p_{6}+p_{1} p_{2} p_{3} p_{4} p_{5}+\right.$

$\left.+p_{1} p_{3} p_{4} p_{5} p_{6}\right)-p_{2} p_{3} p_{4} p_{5}-p_{2} p_{3} p_{5} p_{6}-p_{2} p_{4} p_{5} p_{6}-p_{2} p_{3} p_{4} p_{6}-p_{3} p_{4} p_{5} p_{6}-$

$-9\left(p_{1} p_{3} p_{4} p_{5}+p_{1} p_{3} p_{4} p_{6}+p_{1} p_{3} p_{5} p_{6}+p_{1} p_{4} p_{5} p_{6}\right)-25\left(p_{1} p_{2} p_{4} p_{5}+p_{1} p_{2} p_{4} p_{6}+p_{1} p_{2} p_{5} p_{6}\right)-$

$-49\left(p_{1} p_{2} p_{3} p_{6}+p_{1} p_{2} p_{3} p_{5}\right)-81 p_{1} p_{2} p_{3} p_{4}$.

$a_{1}=\frac{16 G}{\pi w^{2} R} p_{1} p_{2} p_{3} p_{4} p_{5} p_{6}-p_{2} p_{3} p_{4} p_{5} p_{6}-9 p_{1} p_{3} p_{4} p_{5} p_{6}-25 p_{1} p_{2} p_{4} p_{5} p_{6}-49 p_{1} p_{2} p_{3} p_{5} p_{6}-$ $-81 p_{1} p_{2} p_{3} p_{4} p_{6}-121 p_{1} p_{2} p_{3} p_{4} p_{5}$.

$a_{0}=-169 p_{1} p_{2} p_{3} p_{4} p_{5} p_{6}$ 